\title{
Death by Denigration: Psychological, Physiological and Mortality Effects of Ageism
}

\section{Robinson BL*}

South London and Maudsley, NHS Foundation Trust, UK

*Corresponding author: Robinson BL, South London and Maudsley, NHS Foundation Trust, UK, Tel: +447779728926; E-mail: Benjamin.Robinson@slam.nhs.uk

Rec date: Nov 28, 2014; Acc date: Dec 22, 2014; Pub date: Dec 24, 2014

Copyright: (c) 2014 Robinson BL. This is an open-access article distributed under the terms of the Creative Commons Attribution License, which permits unrestricted use, distribution, and reproduction in any medium, provided the original author and source are credited.

\begin{abstract}
Most people have the luxury of growing up as members of the majority in society, thus avoiding the stereotype and prejudice inevitably attendant on being part of a minority group. Such a happy lot, however, is merely transitory, for all of us who reach old age-ironically, a vast majority-will join a people for whom that flavour of prejudice and stereotype known as ageism is a daily occurrence. Ageism was first described as "another form of bigotry" (Butler 1969), and more recently defined as creating a situation whereby "the elderly in our society find themselves viewed in a predominantly negative fashion, victims of a pervasive form of discrimination and disparagement". The negative effects of such prejudice on how the elderly are treated by society and on their own self-esteem are only too wellknown, and it is not the purpose of this discussion to restate them once again. Rather, the focus of this essay is on interesting new research which suggests that ageism may go further than the social or psychological, and have serious negative physical effects, including decline in cognitive function (Levy, 1996) and altered autonomic reactivity. Such claims are examined below, beginning with an analysis of the nature of stereotypes of the elderly and then considering how these impact on old people's views of themselves. The discussion then assesses the validity of the proposed links between such views and both cognitive function and cardiovascular response to stress. This thread of research culminates in a recent paper which proposes a link between how the middle aged and elderly view themselves in terms of aging, and mortality up to twenty years later (Levy et al., 2002).
\end{abstract}

Keywords: Denigration; Death; Psychological; Ageism

\section{Introduction}

Most people have the luxury of growing up as members of the majority in society, thus avoiding the stereotype and prejudice inevitably attendant on being part of a minority group. Such a happy lot, however, is merely transitory, for all of us who reach old age ironically, a vast majority - will join a people for whom that flavour of prejudice and stereotype known as ageism is a daily occurrence. Ageism was first described as "another form of bigotry" [1], and more recently defined as creating a situation whereby "the elderly in our society find themselves viewed in a predominantly negative fashion, victims of a pervasive form of discrimination and disparagement" [2]. The negative effects of such prejudice on how the elderly are treated by society and on their own self-esteem are only too well-known, and it is not the purpose of this discussion to restate them once again. Rather, the focus of this essay is on interesting new research which suggests that ageism may go further than the social or psychological, and have serious negative physical effects, including decline in cognitive function [3] and altered autonomic reactivity [4]. Such claims are examined below, beginning with an analysis of the nature of stereotypes of the elderly and then considering how these impact on old people's views of themselves. The discussion then assesses the validity of the proposed links between such views and both cognitive function and cardiovascular response to stress. This thread of research culminates in a recent paper which proposes a link between how the middle aged and elderly view themselves in terms of aging, and mortality up to twenty years later [5]. This startling proposal - that ageism can contribute to death by causing a low self-esteem about being old - is examined in detail and forms the main thesis of this discussion. Such an analysis is essential, for if the claim is valid, then a new avenue for therapeutic intervention becomes apparent. The nature of such intervention forms the final part of the discourse, the underlying message of which is that society must acknowledge the profound effect it has on the lives and deaths of its elderly. This message is not new, but the following analysis of novel research may imbue in it a much-needed urgency to combat denigration of the elderly. From such pressing need the discussion proceeds.

\section{From cradle to grave: stereotypes of the elderly}

Whilst the elderly are seen as having some positive qualities, negative views about them predominate in Western society. A metaanalysis of studies investigating how people see the elderly shows a 0.38 effect size in the direction of more negativity [6]. The reader will themselves be familiar with stereotypes of senility and decline reinforced daily on television, in books, in magazines and in conversation. Commonly the elderly are seen as more forgetful, withdrawn, defensive, confused and unhappy than younger persons [7] In order to understand the potential effects on the elderly person of such persistent stereotypes, and therefore prevent or ameliorate these, it is necessary to understand how they develop.

Stereotypes of the elderly are acquired when one is young [8]. Therefore, they are internalised and cemented long before an individual becomes part of the elderly community to which they pertain [2]. Other forms of prejudice are not developed independently of reality in this way. Black Americans, for example, encounter stereotypes against themselves as the elderly do, but develop strong group identities at the same time. They are thus able and motivated to analyse the validity of the stereotypes by comparison with their own experience. Since, as mentioned, stereotypes of the elderly develop 
Page 2 of 6

when young, there is nothing real - no group identity - with which to compare them, and they are therefore far more likely to be accepted without questioning. They fall into that category of beliefs which one accepts unconditionally, unaware of any alternatives, and known in the literature as premature cognitive commitments [6]. The power of such beliefs is that once accepted, they freeze an individual's understanding of the belief, preventing it from being reconsidered. A child reading a Fairy Tale depicting a decrepit, forgetful, malicious old person may form such a premature cognitive commitment, which acts as a strong foundation upon which the child assimilates further information about the elderly. Research suggests that children develop such views of the elderly by the age of six [8]. In this way, society promotes and perpetuates negative views of the elderly in the young, such that when these young become old, they already have robust prejudices about what being old is like. Such stereotypes do not, of course, affect how one views oneself whilst young. However, when old, stereotype and self-perception will come into conflict, as selfperception gradually comes to mean not just how one sees oneself as a person, but how one sees oneself as an old person. In this way, when society's prejudices become self-relevant, they will affect the selfperception of the old person [6].

Ageism differs from other forms of prejudice not just in how it develops but in how it impacts on daily life. For racism, this impact is viewed as a function of particular circumstances which emphasise the stereotype. This idea is known as stereotype threat. Members of stigmatised groups experience stereotype threat only when they are in situations in which other people may view them stereotypically. Thus, an individual's stereotype becomes important only when that individual is asked to perform a task which, according to the stereotype, he or she will do badly, and high performance pressures result [5]. For example, and common in the United States, Black Americans are viewed stereotypically as achieving less academically. A Black American, well aware of this stereotype, will experience stereotype threat when his academic prowess is challenged by some task. Often, stigmatized groups protect themselves by avoiding situations which engender stereotype threat. Thus, for the Black American the stereotype threat is focused on academic performance, and is relatively easily, if sadly, avoided by withdrawing from the academic domain. For an elderly person, by contrast, the stereotype is far more diffuse, relating to general health and its absence, to declining social functions - it is of "a diffusiveness that transcends domains" [5]. This is because the underlying theme of the stereotypes is failing health and, ultimately, death. Thus, the nature of the ageist stereotype renders avoidance of stereotype threat impossible. This means that old people cannot help but be subject to daily challenges to their self-perception by activation of stereotypes [9-12]. This, in addition to the unquestioned internalization of age stereotypes in childhood described above, renders the stereotype-threat model - which assumes explicit, occasional, situation specific challenges, inapplicable to the diffuse and pervasive prejudice encountered by the elderly. This is important, for it means they do not have the option for retreat and avoidance of stereotypes available to other stigmatized groups.

In summary, stereotypes of the elderly are internalized whilst the person is still young, and are therefore particularly difficult kinds of prejudice to change. Further, they are wide-ranging, touching on all aspect of life and impossible to avoid. The next section examines experimental evidence that such stereotypes, when they impact upon perception of the self, have the power to alter cognitive and physiological parameters.

\section{Power over heart and mind: Self-stereotypes, memory, and cardiovascular response to stress}

The contention that stereotypes have significant physical effects on old people is relatively novel. Thus, whilst there is evidence to support this claim, it is not extensive and may not be applicable to real life. One reason for this latter problem is the difficulty in designing an experiment which demonstrates a link between negative stereotypes and negative physical effects. Such an experiment would have to activate a long-held prejudice, measure the effects of this activation on various parameters of function, and apply these results to real life. In relation to this last point, previous research has shown that stereotypes of the elderly can be activated and affect perception of old people without entering conscious awareness; indeed, it is generally believed that much prejudice operates subliminally [2]. Thus, any overt, explicit attempt at prompting participants in a trial to call to mind stereotypes of old people would be grossly different from the subliminal activation which occurs in everyday life. In addition, participants may give responses to questions such as "describe this elderly person" that are more socially acceptable than accurate. For this reason, the experiments outlined below attempt to activate stereotypes subliminally, thus better emulating real life and avoiding problems attendant on participants attempting to 'please' researchers [13]. These challenges are mentioned in order both to explain the complexity of the methods outlined below, and to infuse a sceptical hue to the reader's assessment of their results. Thus, the following discussion does not aim to be a forceful argument based on weak evidence, but an early assessment of research that may one day be of proven significance. The justification for such an early analysis is that if the results below are replicated, they will have profound implications for how society treats its elderly.

Let us now move on to the few studies examining this possible link between stereotypes of aging and physical processes. As noted above, one persistent stereotype is that the elderly are forgetful, suffering inevitable memory loss. Currently, most people and most researchers believe that there is an inevitable memory decline with age. However, some studies suggest that perhaps the decline is not normal and may be preventable. In this latter camp, Levy et al. [3] hypothesised that perhaps the stereotypes about aging themselves might result in decreased cognitive function. To investigate this possibility, the researchers flashed old- age relevant words on a screen slow enough for unconscious experience to occur but too fast for conscious awareness. This process, known as 'priming', has been shown to be effective in several prior studies by different groups. Ninety individuals above sixty years of age were recruited by advertisement, and comparison was made with their performance on various memory tasks before and after priming with either positive or negative subliminal messages about aging. The positive prime included words such as accomplished, alert, creative, enlightened, guidance, sage and wise, whilst negative words included Alzheimer's, confused, decline, diseases, incompetent, misplaces and senile. In each group these words were flashed up on screen for 55 milliseconds. The performances on short-term memory tasks - which included photo recall, shape recall, and auditory recall-were compared between the two groups before and after priming. For immediate recall, the mean score was 4.44 out of seven before negative words were flashed up, and only 2.67 after this priming-a significant decrement (paired t test, $\mathrm{t}(44)=6.61, \mathrm{p}<0.01$ ) $[14,15]$. This result is striking when compared to the effect of positive priming, which resulted in a change from 3.40 before priming to 4.38 afterwards-a non-significant increase. Other memory scores showed similar changes, with positive age-associated words causing 
increments in memory scores, some of which reached significance, and negative words causing significant decrements.

These results support the proposal that activated negative stereotypes of aging do affect cognitive function as measured by memory tests. To test whether the effect was due to activation of selfstereotypes rather than, for example, low mood caused by negative words, two further measures were conducted. Firstly, to check that the word-flashing affected old people's views of the elderly, a story was read involving an old person, Margaret, engaged in various activities which could either be interpreted as negative or positive [16-19]. This negative-positive axis was defined in three dimensions: dependentinterdependent, loss of reality-creativity, and forgetting due to senility versus forgetting due to other causes, such as lack of sleep. For example, Margaret's seeing shapes in clouds could either be viewed as creative or losing touch with reality. The participants view of Margaret in both groups (negative and positive priming) was assessed; as hypothesised, in the group which had seen negative words flashed, Margaret's actions were seen as much more dependent, out of touch with reality, and senile when compared with the positive assessment of the other group. Secondly, to ensure that the effects observed were due to activation of self-stereotypes, the whole study was repeated using 90 young people, and this showed no stereotype-induced memory changes, indicating that these stereotypes were activated and are powerful only if self-relevant.

Whilst these results support the possibility that negative selfstereotypes of aging both alter old people's perceptions of aging and affect their cognitive function, its limitations are obvious. The study was an artificial simulation of reality, used a small sample size, and measured changes only in the very short-term. However, it does show that stereotypes do have some link with cognition in the laboratory, and so they might have in real life. As mentioned, this field of research is too novel for any further assessment at this stage. Let us therefore move on from 'power over the mind', to paraphrase this section's subtitle, and examine 'power over the heart' - namely effects on cardiovascular function.

Research on racism has shown that Black Americans subjected to 'stereotype threat' (see Part 1) before carrying out a stressful task, display heightened cardiovascular responses to that task, when compared to colleagues performing the same task in the absence of prior stereotype threat. In the light of this research, one study examined the effect of self-stereotypes of aging on cardiovascular response to stress in the elderly [4]. Here, the priming was used as in the memory and stereotype research. Fifty-four people over sixty years of age were primed either positively or negatively with relation to aging using subliminally flashed words described above. Experimenters measured systolic and diastolic blood pressure, heart rate, and skin conductance before any intervention, after a priming period, and after a mathematical stressor involving counting back from 956 in sevens "as quickly and accurately as possible".

This study found an increase in systolic blood pressure in response to stressful tasks after negative priming, and a reduction in systolic blood pressure in response to the stressor following positive priming. Unfortunately, the raw figures, or even means, are not available - data is only included after statistical analysis assessing significance, or in graph form. From the graphs, however, it appears as if systolic blood pressure, for example, increased by $8 \mathrm{mmHg}$ (95\% confidence interval: +3 to $+12 \mathrm{mmHg}$ ) for the negative priming group, and decreased by 5 $\mathrm{mmHg}$ for the positive group (95\% confidence interval:- 1 to -10 $\mathrm{mmHg})$. These changes are described as significant $(\mathrm{p}<0.05)$. As far as assessment is possible, therefore, this research extends the range of effects of self-stereotypes from the cognitive to the cardiovascular, providing some evidence that the response to stress is altered by activation of self-stereotypes.

These results are consistent with the contention that stereotypes may negatively alter parameters of cognitive and autonomic function. If they are applicable to real life, they hint at a process whereby elderly people, with long-held preconceptions of how poorly society thinks they should function, function less well as a result [20]. Thus, their own low expectations result in a lower level of functioning. This possibility is supported by a further parameter measured in both these studies, namely self-rated efficacy. If low expectations do result in poorer function, this parameter should be low with negative primes and high with positive ones. Thus, all participants were asked to rate how well they thought they would do in mathematical, verbal and memory tasks, before being asked to do them, but after priming. In all cases, those primed negatively, with 'senile' cues, rated themselves as significantly less good than those primed with 'wise' cues. And, as mentioned above, they did indeed perform less well. This supports the hypothesis that the negative stereotype primes activated negative selfstereotypes, which then lowered expectations about performance and therefore performance itself. In other words, the mediator between negative stereotypes and negative effects may be negative assessment of one's own abilities [21]. Thus the memory decline so often ascribed to natural aging may in part be the result of a self-fulfilling prophecy, a cycle whereby prejudice creates the very stereotypes it describes, reinforcing itself with each generation. This possibility is just as significant for the second study, where low expectations, by hampering performance, would increase frustration and hence the stress response. Thus, ageism may itself causes chronic heightened cardiovascular responses to stress. This is particularly interesting in the light of research linking repeated acute blood pressure elevation and the development of chronically elevated blood pressure.

These are heady, not to say premature, claims, and before discarding the cornucopia of medications to treat heart disease-and nowadays memory loss as well-it is worth reapplying that sceptical hue mentioned above, and putting the research in context. These studies are lonely explorers in a relatively uncharted new world, and have yet to prove reproducible by researchers other than Levy et al. Other serious drawbacks include small sample sizes, small effects, artificial laboratory conditions designed to simulate complex life experiences, and failure to include raw data in the results [22]. The above hypothesising will appear at best precocious if the next hundred studies fail to show an association between subliminally introduced images and psychological or physiological effects.

However, there is something challenging, if not downright unsettling, about these hints that our prejudices against the elderly may have such profound cognitive and physical effects upon them. If there is a link between ageism and cognitive decline, we must accept that there would be less decline in elderly people living in a non-ageist society. This presents an obvious avenue of further research, and in lieu of a time machine and unbounded optimism, researchers have looked to two of today's societies to test this idea: China, and the American Deaf Community [6]. It is to the results of this research that the discussion now turns, in an attempt, perhaps, to prove the denigration-decline theory false and ameliorate the feelings of unease which it generates.

It is well known that the Chinese have traditionally respected their elders. Indeed, for the two thousand years before 1949, practices 
Page 4 of 6

displaying respect for or helping the aged were officially endorsed by the government, based as they were on the widely supported ideas of Confucius. The expected disappearance of such practices and reduced respect for the elderly attendant upon the coming to power of the antiConfucian Communist government in 1949 did not materialise. Indeed, there is evidence to show that traditional views of old age have become even stronger in the last fifty years [23]. As for the Deaf community, anthropological studies show that American Deaf adults of all ages identify and interact with each other far more than adults of the mainstream American community, and meet several times each week at social clubs. Interestingly, the young of Deaf communities often treat older Deaf adults as wise leaders and role models. Levy and Langer performed a cross-sectional study examining the views of aging and memory performance of old and young members of the mainstream American, Deaf American, and Chinese (Peking) communities [6]. It will be noted that a more conclusive study would be longitudinal, measuring decline as people from the different communities aged. Levy and Langer compared memory scores in the young and old as a proxy measure for repeated memory tests in one generation as this cohort aged over time. They therefore assumed, but did not know, that differences were due to decline of the elderly rather than different abilities of different generations. However, since the Deaf and Chinese communities are different in almost every respect save for their attitude to the elderly, it would be unlike for the same generational differences to occur in both, providing a degree of control for generational effects. Thus, the study is a good albeit imperfect response to the hypothesis that the elderly of non-ageist communities should show decreased cognitive decline compared with those of ageist communities. As with studies previously presented in this discourse, sample size was small ( $n=90$, with 30 people from each community). Importantly, however, the participants from different communities were matched by age, sex, social class and years of education, so that these effects could be controlled for.

First, the researchers established differential views of aging amongst the three cultures, measured using accepted questionnaires on the subject.

\begin{tabular}{|l|l|l|l|}
\hline Age Cohort & Hearing & Deaf & Chinese \\
\hline Young & 1.74 & 3.93 & 4.26 \\
\hline Old & 2.90 & 4.53 & 5.55 \\
\hline
\end{tabular}

Table 1: Mean scores for positive view of aging (range -1.49 to 7.41 ).

These results show that the young and old Deaf and Chinese cohorts had, as expected, significantly more positive views than the hearing Americans $(\mathrm{p}<0.1)$, the Chinese having most positive views. Next, the researchers used the $7 / 24$ memory task, whereby the participants had to remember the position of dots on a grid, immediately after seeing the pattern and half an hour later. This task was chosen since it can be done even with poor motor function and limited eyesight.

\begin{tabular}{|l|l|l|l|}
\hline Age Cohort & Hearing & Deaf & Chinese \\
\hline Young & 1.69 & 0.98 & 1.34 \\
\hline Old & -2.97 & -1.55 & 0.5 \\
\hline
\end{tabular}

These results show that there is no significant difference in the averaged scores on various memory tests for the young of the three communities. For the old, however, a significant culture effect emerges $(\mathrm{p}<0.01$ by analysis of variants (ANOVA)). The Chinese and deaf elderly performed significantly better on the 7/24 memory task than their hearing American counterparts.

Further analysis shows that the greatest difference is between the Chinese cohort and the two American groups. As shown in Table 1, it was the Chinese who had the most positive view of aging. These results, therefore, all support the proposal of a link between societal views of aging and subsequent cognitive decline. Amongst the Chinese and Deaf communities, where views of aging were more positive, old people outperformed their American counterparts on memory tests. It is particularly striking that in the Chinese participants, there was no significant difference between the memory scores of the young and the old (Table 2). Thus, this research suggest a strong social-psychological component to memory retention in old age, indicating that the memory loss which many of us assume to be inevitable is in fact simply a product of that very belief.

To summarize the evidence examined so far, in support of the hypothesis that socially accepted views of the elderly themselves cause physical and cognitive decline, this discourse has examined small, laboratory-based investigations which use sample sizes of between 50 and 150. These have shown a possible link, with altered cardiovascular response and decreased cognitive performance in response to age stereotypes, and in addition, have demonstrated less cognitive decline in less ageist communities. Whilst interesting, these studies are of a size and nature that is hypothesis-forming rather than effect-proving. How then, to further the analysis? At the start of this discussion, it was noted that stereotypes of the elderly are particularly difficult to avoid since they are based on a fear which touches on every aspect of later life: the fear of death. And it is to death we must now turn, for if society's views on the elderly lead to reduced cognitive function and deleterious cardiovascular effects, then it must be expected that they will ultimately impact upon the ultimate, increasing mortality in those negatively affected by them. This is a logical extension of the results described above, and would give them more weight if found to be the case [24]. Furthermore, showing a mortality effect would lift research on self-perception out of the small-scale laboratory based realms which have been so far been discussed, into the realms of real-life and real importance. The next section therefore examines a study which tries to answer the question of whether 'death by denigration,' the titular claim, does indeed exist.

\section{Power over life and death: Self-perception and mortality}

As any reader of glossy magazines will know, and any physician treating said reader will rue, the number of potential risk factors for developing disease and for dying are lesion. They range from wellaccepted physical factors such as smoking and heredity, to hotly disputed psychological factors such as personality, life events, andnow, self-perception of aging. Most importantly, by no means all researchers accept the validity of the assumption that psychological factors can have mortality effects. It is apparent that for such observers, the status of another putative social-psychological "risk factor" is very low indeed. This section argues first that psychosomatic effects are real, and then that self-perception of aging itself has a mortality effect.

Much research on psychosomatic effects attempts to correlate psychological parameters and life events with later disease course or 
development. Another approach, as described above, is to simulate in the laboratory a psychological state proposed to occur in real life (the negative effect of age stereotypes) and measure resulting changes to parameters (memory scores, blood pressure) which it is hoped correlate with real life pathologies. Both approaches are flawed, in terms of small sample size and dubious relationship to real life, leading some observers to claim that "our belief in disease as a direct reflection of mental state is largely folklore". Another research tradition, however, is more difficult to dispute. This relies on the notion that the timing of death might be altered by psychological factors. Using the anticipation of a festival as this psychological factor, one study. Examined the death statistics for Jewish men around the time of Passover, the most attended Festival in American Jewish culture. The number of deaths (i.e. sample size) was 1919, much larger than usual for psychosomatic research, and a significant drop in death rate was evident in the weeks leading up to Passover, with a corresponding peak just afterwards. A non-Jewish control group had no such dippeak effect. This result was not explicable by general trends in death rates, nor time-of-month (the Jewish calendar is lunar). Only two explanations seemed plausible: that 'will-to-live' - high around festival time - is associated with reduced mortality, and that social occasions have beneficial impact on disease. The results were replicated in Chinese women $(n=1288)$ around the important Harvest Moon Festival and found in the general population around the turn of the second millenium. Here, then, is good evidence that psychological factors can have effects on mortality. Accepting this possibility, let us move on to examine whether one of those factors could be selfperception of aging.

In 1975, Atchley and colleagues recruited almost every person over 50 years of age living in the small town of Oxford (in Ohio, that is - the English version of the town having, perhaps, already exhausted its elderly with research). 1157 participants (total town population=15 000) were thus recruited, and the Ohio Longitudinal Study of Aging and Retirement (OLSAR) begun, with the general purpose of investigating the psychology of aging. Levy and colleagues [5] used data from this study and mortality records to examine whether selfperceptions of aging measured in 1975 correlated with mortality between 1975 and 1998. The OLSAR measure of self-perception of aging was the Attitude towards Own Aging subscale of the Philadelphia Geriatric Center Morale Scale (PGCMS). This consists of the following items: "Things keep getting worse as I get older", "I have as much pep as I did last year", "As you get older, you are less useful", "I am as happy now as I was when I was younger", and "As I get older, things are (better, worse, or the same) as (sic) I thought they would be."

To analyse the results, self perceptions of aging scores were dichotomized into 'high' and 'low', and used as a variable in a KaplanMeier survival analysis - a plot of probability of being alive against years after the 1975 baseline. The 'low' self-perception group fell to the 0.5 survival probability 15 years after 1975 , whilst the 'high' group fell to this level only after 22.6 years. Thus, the median age of survival in the more positive self perceptions of aging group was 7.6 years longer than those in the less positive group. The two groups were significantly different $(\mathrm{p}<0.01)$.

This is a striking result - if true, it would put low self-perception of aging as a greater risk factor than high blood pressure, cholesterol, smoking, poor, diet, and loneliness. Let us examine the claim more carefully. The authors suggest that self-perception is an independent risk factor for death. However, 'low self perception' may result from another cause of mortality, not be the cause itself. After all, an elderly person with two previous myocardial infarctions must surely have a dimmer view of their own aging than a healthy person, and will die sooner. This does not mean that their mortality is linked to their selfperception. In order to account for this, the authors attempted to control for health. The original OLSAR survey included a six-item functional health measure, which asked questions such as "are you able to walk up a flight of stairs?," and "are you able to do heavy physical work?". Unfortunately, this analysis did not include other more objective health measures such as physician assessment or data on previous medical history. Habits such as smoking, alcohol consumption and lifestyle were also not assessed. This control was therefore inadequate, calling into question the validity of the results. Other potential confounders - namely age, gender, and socioeconomic status, were, however, controlled for, and the relationship between higher mortality and lower self-perception was significant for the young (less than 60 ), the old, men, women, the poor, and the rich.

This mixed picture - a seemingly very strong association but with inadequate controls - means that any putative causality between selfperception of aging and mortality is unproven by this study. Standard epidemiological criteria for causality are only partly satisfied. Thus, the strength of the relationship is unclear due to several potential confounding factors, but there does seem to be a definite temporal relationship. In addition, there may be a dose-response relationship: when analysed as a continuous variable, it was found that the lower the self-perception, the higher the risk of mortality. Another important criterion for causality is that of a biologically plausible explanation. Clearly, any putative relationship between self-perception of aging and mortality is difficult to entertain without such an explanation, the candidates for which are discussed below.

Extending the work of Phillips and King described above, Levy et al., in a second study in the same paper [5] propose that 'will-to live' has a more general role than staving off death around the time of festivals. Defining will-to-live as "the judgement that the perceived benefits of ones life transcend the perceived hardships," the authors suggest that low self-perception about one's own aging leads to a lower will-to-live. At some point, when the hardships outweigh the benefits, this will-to-live becomes negative - a will-to-die. It is possible that such a will-to-die partially explains the data of Levy et al. In fact, the original OLSAR data included a will-to-live item, consisting of paired words at either end of a seven-point scale - namely: empty-full, hopeless-hopeful, worthless-worthy. Levy et al. found that this measure did correlate both with self-perceptions of aging and mortality, supporting the will-to-live hypothesis. The researchers therefore describe will-to-live as the 'mediator' between low selfperceptions of aging and mortality. However, it is clear that will-to-live is an inadequate explanation in terms of biological plausibility, since it provides no mind-body association. Even though most scientists have abandoned Cartesian Duality, this is no excuse for abandoning the need to explain how the mind actually affects the body. Will-to-live merely provides a further psychological mechanism for the results. More promising, perhaps, is recent psychoneuroimmunological theory. In a study mentioned above [4] a possible link was shown between self-stereotypes and the stress response. One plausible explanation would therefore be that negative self-perception of aging is a stressor and therefore, like stressful life events, can have negative cardiovascular repercussions, leading to morbidity and early mortality. This theory remains to be tested, but at least shows that the link is compatible with current thinking. 
Page 6 of 6

To summarize, Levy et al's work provides a fascinating suggestion that self-perception of aging might have a significant mortality effect. This is especially important because here is a potential risk factor determined to a large extent by society's views of the elderly. But the research is not wholly depressing, for there are two messages that arise if these data are replicated: the discouraging one is that negative self perceptions lead to earlier mortality; the encouraging one is that positive self-perceptions prolong life expectancy. As noted, however, this is new research which does not fulfill the epidemiological criteria necessary to establish causality. In terms of future direction, therefore, the main discussion at this stage should be about further research aimed at establishing the association, rather than therapeutic interventions based upon it. Nevertheless, the issue of therapy will be touched upon in the next and final section- with little more justification, perhaps, than the excitability of youth (language, it will be noted, has an ageism of its own.)

\section{Future research and potential therapy}

The research that inspired this discussion relied on data that had been measured for a separate study twenty years earlier. Thus, the obvious next step is to instigate a longitudinal cohort study which measures self-perception of aging at a point in time, and repeats these measures at regular intervals, concurrent with collecting mortality data. Ideally, such a study would be replicated in several different cultures with different attitudes to the elderly. Such a prospective, longitudinal design would avoid the problems outlined above, where the authors were unable to choose which parameters to measure and hence control for. Another strand of research should focus on potential biological explanations for the link between the socialpsychological mechanism discussed in this essay and the somatic effects measured in the studies. This would help strengthen the argument that such a link exists.

If the link were supported, what then of intervention? Since the ultimate cause of low self-perceptions of aging are the stereotypes of the elderly internalised when young, the way to prevent old people having such self-perceptions is to alter how society views its elderly. This would require a society, like the Deaf in America, where the young have much more contact with the elderly, so that positive relationships can be built up and reality can begin to replace stereotype. Doctors, in daily contact with the elderly, should be some of the first to promote realistic views about them. In addition, doctors could balance the negative views of society by informing the elderly of research showing that memory loss and physical decline may not be inevitably attendant on aging. However, the bottom line is that the intervention which follows from this discussion would require a concerted society-wide effort to eradicate ageism - a challenge indeed.

In summary, then, the putative link between mortality and selfperception of aging needs better evidence to support it, but if true, offers immediate and obvious therapeutic intervention. Unfortunately, any implementation requiring an altering of society's views is, to put it mildly, difficult. After all, it is no new concept that ageism is wrong, nor that the elderly might benefit from more interaction with the rest of society - yet views and actions remain as prejudiced as ever. This discussion has presented promising research, still in its early stages, which suggests that such views might have serious physical effects, including earlier mortality. If these data are replicated, they may at last provide the spur that society needs to end the damaging denigration of the elderly which it currently perpetrates.

\section{References}

1. Butler RN (1969) Age-ism: another form of bigotry. Gerontologist 9: 243-246.

2. Perdue CW, Gurtman MB (1990) Evidence for the automaticity of ageism. Journal of Experimental Social Psychology 226: 199-216.

3. Levy B (1996) Improving memory in old age through implicit selfstereotyping. J Pers Soc Psychol 71: 1092-1107.

4. Levy BR, Hausdorff JM, Hencke R, Wei JY (2000) Reducing cardiovascular stress with positive self-stereotypes of aging. J Gerontol B Psychol Sci Soc Sci 55: P205-213.

5. Levy BR, Slade MD, Kunkel SR, Kasl SV (2002) Longevity increased by positive self-perceptions of aging. J Pers Soc Psychol 83: 261-270.

6. Kite ME, Johnson BT (1988) Attitudes toward older and younger adults: a meta-analysis. Psychol Aging 3: 233-244.

7. McTavish DG (1971) Perceptions of old people: a review of research methodologies and findings. Gerontologist 11: Suppl: 90-101.

8. Isaacs LW, Bearison DJ (1986) The development of children's prejudice against the aged. Int J Aging Hum Dev 23: 175-194.

9. Levy B, Langer E (1994) Aging free from negative stereotypes: successful memory in China and among the American deaf. J Pers Soc Psychol 66: 989-997.

10. Angell M (1985) Disease as a reflection of the psyche. N Engl J Med 312: 1570-1572.

11. Barnett PA, Spence JD, Manuck SB, Jennings JR (1997) Psychological stress and the progression of carotid artery disease. J Hypertens 15: 49-55.

12. Clark R, Anderson NB, Clark VR, Williams DR (1999) Racism as a stressor for African Americans. A biopsychosocial model. Am Psychol 54: 805-816.

13. Banaji M, Hardin C, Rothman A (1993) Implicit stereotyping in person judgements. Journal of Personality and Social Psychology 65: 272-281

14. Becker G (1980) Growing old on silence. Berkely: University of California Press.

15. Blascovich J, Spencer SJ, Quinn D, Steele C (2001) African Americans and high blood pressure: the role of stereotype threat. Psychol Sci 12: 225-229.

16. Crocker J, Major B, Steele C (1998) Social Stigma. In D.T. Gilbert, The handbook of social psychology pp. 504-553, New York: McGraw Hill.

17. Davis D (1983) Long lives: Chinese elderly and the Communist revolution. Cambridge, MA: Harvard University Press.

18. Johansson B, Zarit SH, Berg S (1992) Changes in cognitive functioning of the oldest old. J Gerontol 47: P75-80.

19. Lawton MP (1975) The Philadelphia Geriatric Center Morale Scale: a revision. J Gerontol 30: 85-89.

20. Phillips DP, King EW (1988) Death takes a holiday: mortality surrounding major social occasions. Lancet 2: 728-732.

21. Phillips DP, Smith DG (1990) Postponement of death until symbolically meaningful occasions. JAMA 263: 1947-1951.

22. Sher A (1984) Aging in post-Mao China: The politics of veneration. Boulder, CO: Westview Press.

23. Sinard JH (2001) Y2k revisited: a human component? JAMA 285: 1706-1707.

24. Wheeler SC, Petty RE (2001) The effects of stereotype activation on behavior: a review of possible mechanisms. Psychol Bull 127: 797-826. 